\title{
Assessment of the Performance of Reconstruction Processes for Computed Tomography
}

\author{
P. Maréchal ${ }^{\dagger \ddagger}$, D. Togane ${ }^{\ddagger}$, A. Celler ${ }^{\ddagger}$ Member, IEEE and J.M. Borwein ${ }^{\dagger}$ \\ ${ }^{\dagger}$ CECM - Department of Mathematics and Statistics, Simon Fraser University, Burnaby, B.C., Canada \\ $\ddagger^{\ddagger}$ Div. of Nuclear Medicine, Vancouver Hospital and Health Sciences Centre, Vancouver, B.C., Canada
}

\begin{abstract}
This paper deals with the assessment of the performance of reconstruction methods for computed tomography, including Filtered Back Projection and 'entropy-like' methods. For each of these methods, the influence of errors in the measured data on the reconstructed image is analyzed. A small perturbation of the data vector induces a perturbation of the reconstructed object which can be computed by means of the sensitivity matrix. Using appropriate matrix computation techniques, an upper bound on the size of the reconstruction error is determined, as well as the pattern of noise in the sinogram that will result in the largest reconstruction error. Simulations will illustrate our analysis and demonstrate its utility in the interpretation of computed images and in the selection of reconstruction parameters.
\end{abstract}

\section{INTRODUCTION}

The reconstruction of images from measurements of radiation around the body of a patient have been extensively studied in the past decades, and many methods have been proposed (see [1] and references therein). All of these methods must find solutions for two major classes of problems. The first one arises from the fact that the relationship between the object to be reconstructed $\phi$ and the physical quantity to be measured $\psi$ is theoretically known but usually undetermined in practice ${ }^{1}$. The second class of problems consists in reconstructing an image from noisy, blurred and under-sampled data.

For several imaging techniques, such as $\mathrm{X}$-ray tomography or SPECT, the simplest model of forward relationship involves the Radon transform or some of its generalizations (attenuated Radon transform, X-ray transform, etc.). We shall write symbolically " $\psi=\mathcal{R} \phi$ ". This (linear) equation can be shown to be the integral form of the so-called transport equation [1], when no scattering is to be accounted for. In spite of recent progress [2], inversion of the transport equation and implementation of suitable regularization schemes for it remain open problems, even if one assumes perfect knowledge of the attenuation map and phase function. Nevertheless, we believe that the mastery of the inverse problem corresponding to its simplified formulation is a necessary step towards a deeper understanding of the problem in its full complexity.

The purpose of the present paper is to introduce some

\footnotetext{
${ }^{1}$ For example, in the case of SPECT, $\phi$ is the distribution of radioactivity, $\psi$ is the outgoing radiation as a function of an angular and position variables. The relation between these distributions involves effects such as the attenuation and scatter, which can not be determined with perfect accuracy.
}

concepts from applied mathematics and to demonstrate, through simulations, how these concepts can provide useful information on the stability and fidelity of reconstruction processes. Before introducing a general framework for the regularization of Radon-type inverse problems, we shall glance at the standard FBP algorithm. The main reason for this is that, unlike heuristic methods, the FBP method allows a rigorous error analysis to be performed.

\section{OVERVIEW OF THE FILTERED BACK PROJECTION METHOD}

Among the essential features of the FBP method are its linearity and the fact that a certain level of resolution is imposed by means of the filtering operation. Recall that the (attenuation-free) Radon transform $\mathcal{R}$ is defined by ${ }^{2}$

$$
[\mathcal{R} \phi](\theta, p) \equiv \int \phi(\xi) \delta(p-\langle\theta, \xi\rangle) d \xi
$$

As pointed out notably in [3], the FBP algorithm can be derived from the following fundamental relationship:

$$
\left(\mathcal{R}^{\star} g\right) \star \phi=\mathcal{R}^{\star}(g * R \phi),
$$

where $\mathcal{R}^{\star}$ denotes the adjoint of $\mathcal{R}, \star$ is the standard 2-dimensional convolution, $*$ is the radial convolution and $g \equiv g(\theta, p)$ is some smoothing kernel (often chosen independent of $\theta$ ). The adjoint of $\mathcal{R}$ is the Back Projection operator. It is defined by

$$
\left[\mathcal{R}^{\star} \psi\right](\xi) \equiv \int_{S_{1}} \psi(\theta,\langle\theta, \xi\rangle) d \theta
$$

Here, $S_{1}$ denotes the unit circle. Ignoring sampling issues, the FBP reconstruction is defined by

$$
\bar{\phi} \equiv \mathcal{R}^{\star}(g * \psi),
$$

where $\psi$ represents the given Radon transform of the original object $^{3}$. The FBP method therefore essentially consists in applying the adjoint of the Radon transform to smoothed projection data.

The discrete version of Eq. (1) reads $\bar{x}=B \tilde{y}$, where $\bar{x}$ the discrete representation of the reconstructed object, $B$ is the matrix corresponding to the application of the FBP algorithm and $\bar{y}$ is the vector representing the noisy and sampled data. It follows that any perturbation $\delta y$ of the data, will induce a perturbation $\delta \bar{x}=B \delta y$ of the FBP solution.

\footnotetext{
${ }^{2}$ For symbols which are not defined in the text, the reader is referred to the Glossary (Section VIII).

${ }^{3}$ In the discrete implementation of the above formula, due to the conflicting polar and Cartesian underlying representation involved in the formula, the filter must include the so-called ramp filter.
} 


\section{INVERSE PROBLEMS AND RECONSTRUCTION PROCESSES}

Suppose we are interested in an object $\phi_{0}$ (such as an image or a sequence of images), but we only have access to a sampling $y_{0}$ of a transformation $\psi_{0}$ of this object:

$$
\begin{aligned}
& L^{(0)} \longrightarrow L^{(i)} \quad \longrightarrow \Re^{m} \\
& \phi_{0} \longrightarrow \psi_{0}=\mathcal{R} \phi_{0} \longrightarrow y_{0}=\mathcal{S} \psi_{0} .
\end{aligned}
$$

Here, $L^{(0)}$ and $L^{(i)}$ denote the (infinite-dimensional) functional spaces in which the object of interest and its image by the transformation $\mathcal{R}$ are assumed to lie, respectively, and $\mathcal{S}$ is the sampling operator. Equation (2) is referred to as the forward relationship. In image reconstruction, the space $L^{(0)}$ is often chosen as the space of square integrable functions whose support lie in some bounded domain.

The problem consists in reconstructing an approximation of the original object, given an approximation $\tilde{y}$ of $y_{0}$. The difference between $\tilde{y}$ and $y_{0}$ is the experimental error.

For many problems of practical interest, the transformation $\mathcal{R}$ is an invertible linear operator. In general, ill-posedness of these problems is primarily due to the fact that the inverse operator $\mathcal{R}^{-1}$ is not continuous, so that to small variations of $\psi \in L^{(i)}$ may correspond very large variations of its inverse image $\mathcal{R}^{-1} \psi$.

A reconstruction process can be regarded as a function which associates an object $\bar{\phi}$ to each data vector $y$ :

$$
\bar{\phi}=\mathcal{F}(y)
$$

For a reconstruction process to be acceptable, $\mathcal{F}$ should meet certain requirements. Firstly, $\bar{\phi}$ should reproduce the data to within the experimental error (fidelity); secondly, $\bar{\phi}$ should not be too sensitive to fluctuations of the data vector (stability); thirdly, $\bar{\phi}$ should be related to the true object in a way that makes it physically interpretable (legibility); finally, $\bar{\phi}$ should be computable in a reasonable time (computability).

The first two requirements are always partially conflicting. Consequently, a compromise needs to be found. Keeping this in mind, it becomes clear that a reconstruction process should provide, together with a reconstructed object, some information on its fidelity and stability. Although of crucial importance, the last two requirements will not be addressed in this paper. All reconstruction methods discussed further perform reasonably well in terms of legibility and computability.

\section{REgULARIZATION SCHEME FOR COMPUTED TOMOGRAPHY}

In practice, reconstructing the object directly as an element of the functional space $L^{(0)}$ may be difficult or even impossible, simply because elements of $L^{(o)}$ have infinite dimension. The first step in the definition of a regularization scheme therefore consists in choosing an appropriate interpolation basis $\left\{e_{j}\right\}_{j=1}^{n} \subset L^{(o)}$, allowing finite dimensional representation of the object. Otherwise expressed, the object to be reconstructed is confined to a finite dimensional subspace of $L^{(0)}$, and is represented by a vector $x \in \Re^{n}$ :

$$
\phi=\mathcal{E} x \equiv \sum_{j=1}^{n} x_{j} e_{j}
$$

We shall call $\mathcal{E}$ the emulation operator. The interpolation functions $e_{j}$ can be regarded as generalized pixels. The component vector $x$ is constrained by the data vector as shown by the following relationship:

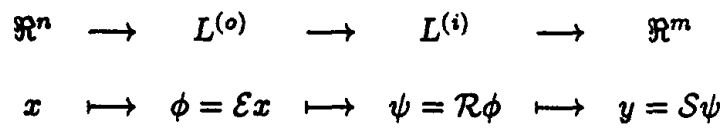

We shall write $R \equiv \mathcal{S R E}$. It can be represented by an $(m \times n)$ matrix. when $\mathcal{R}$ is the Radon transform, the entries of $R$ are given by the following formula:

$$
R_{j, k l} \equiv \mathcal{R}_{j}\left(\theta_{k}, p_{l}\right)=\int e_{j}(\xi) \delta\left(p_{l}-\left\langle\xi, \theta_{k}\right)\right) d \xi
$$

It should be noted that confining the object to $\mathcal{E} \Re^{n}$ may stabilize it, to some extent. In general, however, interpolation bases that are rich enough to allow a convenient representation of the object (in other words, interpolation bases which emulate the original object workspace $L^{(0)}$ well enough) do not sufficiently stabilize the reconstruction.

The second step then consists in defining the component vector of the reconstructed object as the minimizer of a regularized objective function $f$ :

$$
\bar{x} \equiv \operatorname{argmin}\left\{f(x) \equiv \varepsilon(y-R x)+\alpha \varrho(x) \mid x \in \Re^{n}\right\} .
$$

In the definition of $f$, the first term forces the solution to fit the data, while the second term stabilizes it with respect to the data vector. In other words, $\varepsilon$ strives for fidelity, while $\varrho$ (whose negative is often called an entropy) aims at stabilizing the reconstruction. The regularization parameter $\alpha$ controls the relative weight of each function. In practice, $f$ will always be a convex function having a unique minimizer, so that $\bar{x}$ in Eq. (4) is unambiguously defined. Naturally, the reconstructed object is obtained from its components via Eq. (3).

In summary, the main issues regarding the definition of a regularized reconstruction process are:

- the choice of an appropriate interpolation basis;

- the definition of the regularized objective function;

- and the development of numerical tools allowing the control of both the fidelity of the reconstruction and its stability.

For a general discussion on this regularization scheme, see [4], Section 3, for example.

For the purposes of this article, we have chosen the standard gate function set as the interpolation basis, which corresponds 
to the familiar notion of pixel. It forms an orthogonal set (with respect to the usual integral scalar product), and can easily be scaled so as to form an orthonormal basis (of $\mathcal{E} \Re^{n}$ ). Examples of regularized objective functions will be given in the next section. A reasonable and easy to calculate fidelity estimator is provided by $\varepsilon(y-R \bar{x})$. The sensitivity analysis can be performed by taking the (implicit) function defining the reconstruction process and linearizing it around the solution ${ }^{4}$, as is shown in the next Section.

\section{Computational issues}

From now on, we assume that $\varepsilon$ is the squared Euclidean norm:

$$
\varepsilon_{0}(y-R x)=\frac{1}{2}\|y-R x\|^{2} .
$$

We list below some classical regularizers:

$$
\begin{array}{ll}
\varrho_{1}(x) \equiv \sum_{j=1}^{n} x_{j} \ln x_{j}, \quad \varrho_{2}(x) \equiv \sum_{j=1}^{n} x_{j} \ln \frac{x_{j}}{x_{0 j}} \\
\varrho_{3}(x) \equiv \frac{1}{2}\|x\|^{2}, & \varrho_{4}(x) \equiv \frac{1}{2}\langle x, Q x\rangle .
\end{array}
$$

In the definition of $\varrho_{2}$, the $x_{0 j}$ 's are the components of some reference model of the object. In the definition of $\varrho_{4}, Q$ is a positive semi-definite matrix. The functions defined above are often referred to as the Boltzmann-Shannon, the Kullback-Leibler, the Tikhonov and the generalized Tikhonov neg-entropies, respectively, from the names of their inventors.

Our purpose here is not to discuss the argumentation leading to the choice of one particular regularizer or the other (a wide literature is devoted to this subject). Nor is it to discuss the choice of particular interpolation basis. The main objective of what follows is to provide numerical tools for the assessment of the performances of each regularizer, in terms of fidelity and stability.

Recall that the reconstructed object is $\mathcal{E} \bar{x}$, where $\bar{x}$ solves the following optimization problem

$$
\left(P_{0}\right) \quad \min \left\{\varepsilon(y-R x)+\alpha \varrho(x) \mid x \in \Re^{n}\right\} .
$$

Constraints may be included in the above problem. For example, each component of $x$ may be required to be nonnegative, reflecting the non-negativity of the object to be reconstructed ${ }^{5}$. In general, $x$ is constrained to lie in some convex set $C \subset \Re^{n}$ corresponding to the prior knowledge one has of the object. Notationally, this can be incorporated in $\left(P_{0}\right)$ by replacing $\varrho$ with

$$
\varrho_{C}(x) \equiv \begin{cases}\varrho(x) & \text { if } x \in C \\ +\infty & \text { otherwise }\end{cases}
$$

\footnotetext{
${ }^{4}$ This is of course possible provided that $f$ possesses certain smoothness properties.

${ }^{5}$ Depending on the nature of the interpolation basis, the constraint $\phi \geq 0$ may give rise to different constraints on the component-vector $x$. However, these constraints are al ways convex, since they can be written as $x \in \mathcal{E}^{-1} L_{+}^{(0)}$, where $L_{+}^{(0)} \equiv\left\{\phi \in L^{(0)} \mid \phi \geq 0\right\}$.
}

Henceforth, we assume that both $\varepsilon$ and $\varrho$ are twice continuously differentiable on their domain. All objective functions involving $\varepsilon_{0}$ and $\varrho_{i}(i=1, \ldots, 4)$ satisfy this assumption. Notice however that, in general, this assumption does not apply to the constrained case. The reason for this is that, even if $\varrho$ is twice continuously differentiable, $\varrho_{C}$ will not be so in practice.

Recall that, for $\bar{x}$ to solve Problem $\left(P_{0}\right)$, we must have

$$
\nabla f(\bar{x})=-R^{\star} \nabla \varepsilon(y-R \bar{x})+\alpha \nabla \varrho(\bar{x})=0 .
$$

Now, let $\delta y$ and $\delta \bar{x}$ be a small perturbation of $y$ and the corresponding perturbation of $\bar{x}$, respectively. Imposing Condition (5) to $y+\delta y$ and $\bar{x}+\delta \bar{x}$ leads to the following first order approximation:

$$
\delta \bar{x} \simeq S \delta y \quad \text { with } \quad S \equiv\left[R^{\star} H_{\varepsilon} R+\alpha H_{\ell}\right]^{-1} R^{\star} H_{\varepsilon} .
$$

The $(m \times n)$-matrix $S$ is referred to as the sensitivity matrix. Here, $H_{\varepsilon_{0}}$ is the identity matrix and $S$ becomes

$$
S=\left[R^{\star} R+\alpha H_{e}\right]^{-1} R^{\star}
$$

Note that the sensitivity matrix for the FBP method is the matrix $B$ itself (cf. Section II). From Eq. (6), various types of error analysis can be performed. In particular, the (spectral) norm of $S$ will provide an upper bound on the size of the reconstruction error ${ }^{6}$. As a matter of fact we have, for all $\delta y$,

$$
\|\delta \bar{x}\| \leq\|S\| \cdot\|\delta y\|
$$

and equality holds when $\delta y$ is a singular vector corresponding to the highest singular value $\sigma_{1}$ of $S$ (recall that $\sigma_{1}=\|S\|$ ).

Slightly more insightful is the computation of the singular vectors associated with the highest singular values, which we shall call the critical modes of the reconstruction. They can be interpreted as artifact images which may have corrupted the reconstruction. This will be illustrated in the next section.

Finally, on denoting $H_{i}$ the Hessian matrix of $\varrho_{i}$ at $\bar{x}$, we can easily see that $H_{1}$ and $H_{2}$ are both equal to the diagonal matrix whose diagonal entries are the inverses of the components of $\bar{x}$, that $H_{3}$ is the identity matrix and that $H_{4}=Q$. Note in particular that the sensitivity matrices of quadratic regularizers (Tikhonov and generalized Tikhonov) do not depend on the optimal solution. In these cases, sensitivity analysis can be performed a priori (i.e. before actually reconstructing the object).

\section{NUMERICAL EXPERIMENTS}

In this section, the above considerations are illustrated by means of 2D numerical experiments. Systematic study of

\footnotetext{
${ }^{6}$ Note that provided that the interpolation basis is orthonormal, the squared Euclidean norm of $\delta \bar{x}$ coincides with the squared $L_{2}$-norm of $\delta \bar{\phi}$ : for all $\phi=\mathcal{E} x$, we have

$$
\|\phi\|_{2}^{2} \equiv \int|\phi|^{2}=\int|\mathcal{E} x|^{2}=\int \sum_{j=1}^{n} \sum_{k=1}^{n} x_{j} x_{k} e_{j} e_{k}=\sum_{j=1}^{n} x_{j}^{2} .
$$
}


regularization techniques for Radon-type inverse problems is deferred to ulterior publication.

From a given test object $\phi_{0}$, we compute samples of its Radon transform over a $(15 \times 60)$-grid in the $(\theta, p)$-plane. Both $\phi_{0}$ and the corresponding sinogram $y_{0}$ are shown in Fig. 1. In the same figure are shown $(40 \times 40)$-reconstructions using Tikhonov's and Boltzmann-Shannon's regularizers.
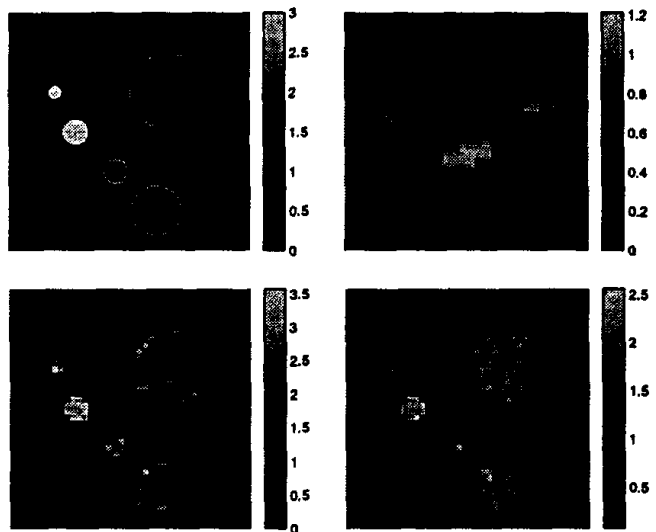

Figure 1: Top left: original object; top right: corresponding sinogram; bottom left: 'Tikhonov' reconstruction, (with positivity constraint) for $\alpha=0.8$; bottom right: 'Boltzmann-Shannon' reconstruction for $\alpha=0.5$.

Figures 2, 3 and 4 show the influence of the regularization parameter $\alpha$ on the fidelity and the stability for both Tikhonov and Boltzmann-Shannon regularizers. The fidelity is defined here as the number $\beta \equiv\|y-R \bar{x}\|$. The stability is assessed according to the analysis presented in Section $\mathrm{V}$. For each value of $\alpha$,

$$
\sigma \equiv\|S\| \frac{\|y\|}{\|\bar{x}\|}=\sigma_{1} \frac{\|y\|}{\|\bar{x}\|}
$$

is computed. The value of $\sigma$ gives the maximum amplification of the relative error, as shown by the following inequality:

$$
\frac{\|\delta \bar{x}\|}{\|\bar{x}\|} \leq \sigma \frac{\|\delta y\|}{\|y\|}
$$

As expected, increasing $\alpha$ causes $\beta$ to increase and $\sigma$ to decrease. The curves displaying $\sigma$ versus $\beta$ can be used in practice for the selection of the regularization parameter. Indeed, $\alpha$ may be chosen so that $\beta$ is approximately equal to the expected norm of the noise ${ }^{7}$.

These curves are also useful for the comparison of the behaviors of different regularizers in terms of the trade-off between stability and fidelity. For a desired $\beta$, the most efficient regularizer is the one for which $\sigma$ is smallest. This doesn't mean that it should necessarily be selected, since other criteria (such as legibility and computability) must be considered.

\footnotetext{
${ }^{7}$ Although $\beta$ clearly depends on each particular data vector, The range of acceptable values of alpha for a given experimental setting is sufficiently narrow, in practice, for a value to be selected once for all.
}
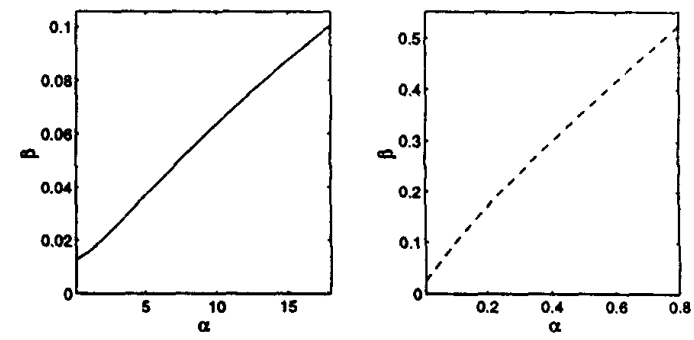

Figure 2: Graphs of $\beta$ (fit) vs. $\alpha$ for Tikhonov (left) and BoltzmannShannon (right) regularizers.
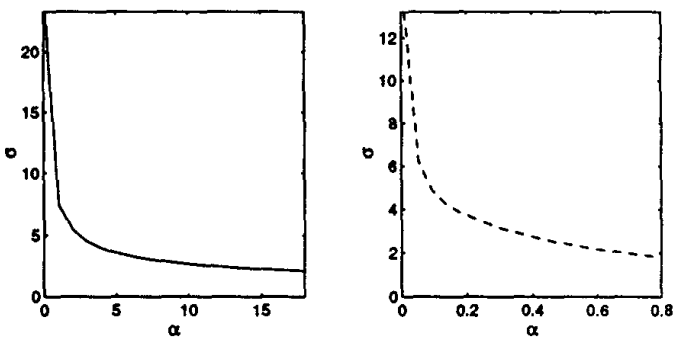

Figure 3: Graphs of $\sigma$ (sensitivity) vs. $\alpha$ for Tikhonov (left) and Boltzmann-Shannon (right) regularizers.

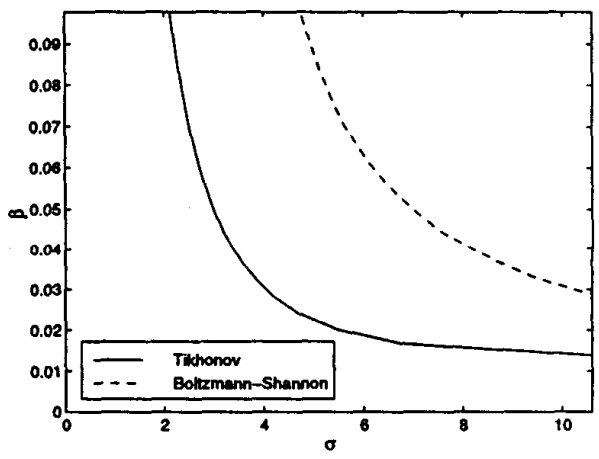

Figure 4: Graph of $\beta$ vs. $\sigma$ for both Tikhonov and Boltzmann-Shannon regularizers.

In Fig. 5, we give an example of error amplification in a case where the problem has not been properly regularized $(\sigma \simeq 13.8)$. The critical perturbation was obtained by computing the Singular Value Decomposition of the sensitivity matrix. This perturbation and the related artifact image correspond to the highest singular value $\sigma_{1}$ (see Fig. 5). Perturbed reconstructions were obtained by adding critical and random perturbations. These perturbations were scaled so as to obtain a relative error of $10 \%$ in the data domain. This illustrates the importance of stability of reconstruction processes.

Finally, Fig. 6 shows caculated values of the norm of the FBP sensitivity matrix for various filters and cutoff frequencies. This graph illustrates the well-known fact that low cutoff frequencies improve stability and therefore reduce the 
(a)

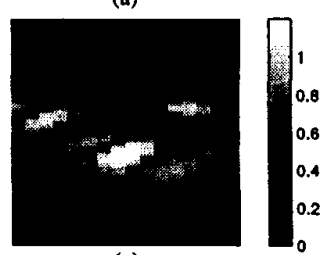

(c)

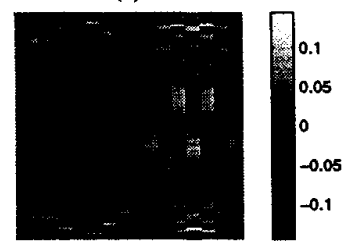

(e)

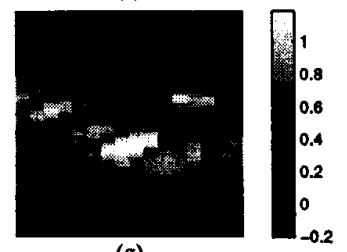

(g)

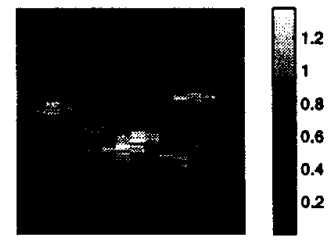

(b)

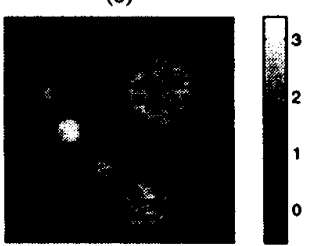

(d)

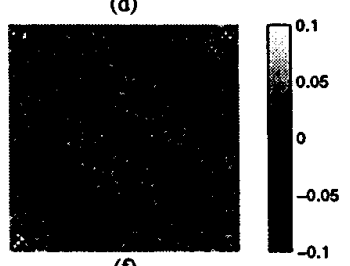

(f)

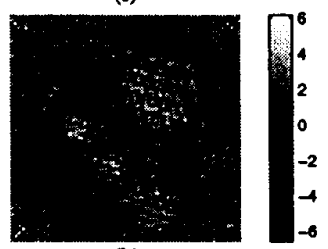

(h)

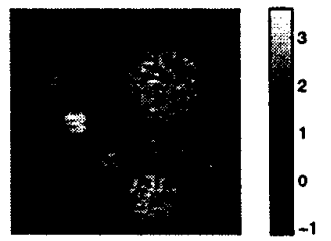

Figure 5: (a) and (b): noise free $(16 \times 96)$-sinogram and $(64 \times 64)$ reconstructed object (using Tikhonov regularizer, with $\alpha=0.5$, so that $\sigma \simeq$ 13.8); (c) and (d): critical perturbation in the data domain and corresponding artifact image; (e) and (f): critically perturbed sinogram and corresponding reconstruction. (g) and (h): randomly perturbed sinogram and corresponding reconstruction.

influence of noise.

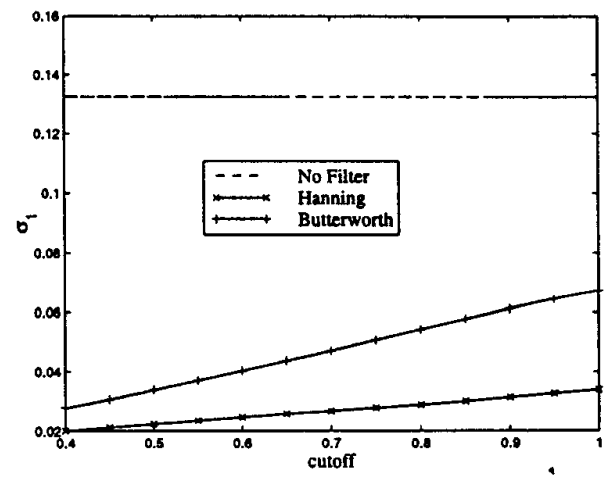

Figure 6: FBP stability vs. filter cutoff frequency.

\section{CONCLUSION}

We have presented a method for assessing the performance of reconstruction processes for Computed Tomography. We have proposed criteria for estimating both the fidelity and the stability of methods (including FBP and methods belonging to the general framework outlined in Section IV). These criteria have been shown to be effective and computationally tractable. This provides quantitative information for the comparison of different methods and allows the informed selection of reconstruction parameters and techniques. In addition, interpretation of reconstructed images will benefit from quantitative information about the reconstruction error.

\begin{tabular}{ll} 
& \multicolumn{1}{c}{ VIII. GLOSSARY } \\
$\mathcal{R}$ & Radon transform \\
$\mathcal{R}^{\star}$ & Adjoint of $\mathcal{R}$ \\
$\mathcal{E}$ & Emulator \\
$\mathcal{S}$ & Sampling operator \\
$L^{(o)}$ & Object workspace \\
$L^{(i)}$ & Image of $L^{(o)}$ by $\mathcal{R}$ \\
$\xi$ & Position variable (object domain)
\end{tabular}

$(\theta, p) \quad$ Angular and position variable (data domain)

$S_{1} \quad$ Unit circle

$\phi \quad$ Generic element of $L^{(0)}$

$\phi_{0} \quad$ Original object

$\bar{\phi} \quad$ Reconstructed object

$\psi \quad$ Generic element of $L^{(i)}$

$\psi_{0} \quad$ Image of $\phi_{0}$ by $\mathcal{R}$

$y \quad$ Generic element of $\Re^{m}$

$y_{0} \quad$ Image of $\psi_{0}$ by $\mathcal{S}$ (ideal data)

$\tilde{y} \quad$ Measured data

$\mathcal{F} \quad$ Reconstruction process

$\left\{e_{j}\right\} \quad$ Interpolation basis

$R$ Forward matrix: $R=\mathcal{S R E}$

$R^{\star} \quad$ Adjoint (transpose) of $R$

$x \quad$ Generic element of $\Re^{n}$

$f \quad$ Regularized objective function

$\varepsilon \quad$ Fit function

$\alpha \quad$ Regularization parameter

$\varrho \quad$ Regularizer

$C$ Convex set representing physical constraints

$\bar{x} \quad$ Minimizer of $f$

$H_{\varepsilon} \quad$ Hessian of $\varepsilon$ at $y-R \bar{x}$

$H_{e} \quad$ Hessian of $\varrho$ at $\bar{x}$

$S \quad$ Sensitivity matrix

* 2-dimensional convolution operator

* Radial convolution operator

$\delta(\cdot) \quad$ Dirac delta function

\section{REFERENCES}

[1] "Mathematics and Physics of Emerging Biomedical Imaging," NRC/IM, National Academic Press, Washington D.C., 1996.

[2] V.G. Romanov, "Conditional stability estimates ...," Sib. Math. J., vol. 35, 1994.

[3] A.G. Ramm and A.I. Katsevich, "The Radon Transform and Local Tomography," CRC Press, 1996.

[4] P. Maréchal, "Sur la Régularisation des Problèmes Mal Posés," PhD. Thesis, Université Paul Sabatier (Toulouse, France), 1997. 